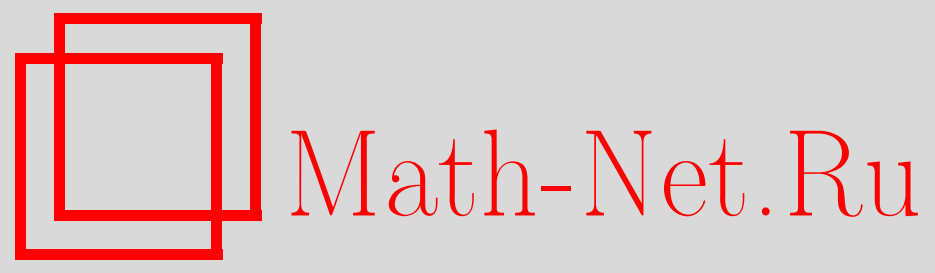

В. А. Колмыков, В. В. Меньших, О переключениях ориентаций графов, Дискрет. матем., 2002, том 14, выпуск $3,18-22$

DOI: https://doi.org/10.4213/dm250

Использование Общероссийского математического портала Math-Net.Ru подразумевает, что вы прочитали и согласны с пользовательским соглашением http://www . mathnet.ru/rus/agreement

Параметры загрузки:

IP: 34.227 .88 .159

26 апреля 2023 г., 17:57:46

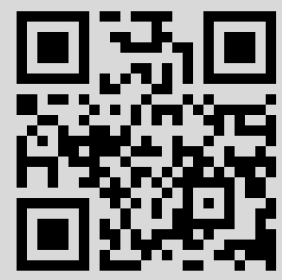




\title{
О переключениях ориентаций графов
}

\author{
() 2002 г. В. А. Колмыков, В. В. Меньших
}

Пусть каждой вершине конечного ориентированного графа сопоставлено конечномерное линейное пространство, а каждой стрелке - линейное преобразование соответствующего пространства. Такие объекты называются линейными представлениями графов. Они естественно возникают в некоторых разделах алгебры и активно изучаются в последние тридцать лет.

Заменяя все стрелки, входящие в вершину-сток на противоположные, получим новый орграф. Эти два орграфа родственны тем, что задачи классификации их представлений, как показали И. Н. Бернштейн, И. М. Гельфанд и В. А. Пономарев, эквивалентны друг другу. Две ориентации называются эквивалентными, если одна получается из другой последовательностью описанных преобразований.

В теории представлений орграфов наиболее интересны бесконтурные ориентации. В этой заметке указывается простой критерий эквивалентности бесконтурных ориентаций. Доказано, что две ориентации эквивалентны тогда и только тогда, когда равны некоторые интегралы от этих ориентаций.

Следуя [1], мы рассматриваем только конечные графы без петель и кратных ребер. Ориентация $\Lambda$ графа $G$ - задание направлений каждого из его ребер.

Для вершины $a$ и ориентации $\Lambda$ через $s_{a} \Lambda$ обозначается ориентация, получающаяся из $\Lambda$ изменением направлений всех ребер, инцидентных $a$. Преобразование $s_{a}$ называется отражением. Для последовательности вершин $A=\left\{a_{i}\right\}_{i=1}^{k}$ положим $s_{A}=s_{a_{k}} \ldots s_{a_{1}}$. Назовем $A$ единицей для $(G, \Lambda)$, если $s_{A} \Lambda=\Lambda$. Последовательность $A$ назовем полной, если она содержит все вершины графа по одному разу.

Лемма 1. Преобразование $s_{A}$ не зависит от порядка, в котором расположены отражения $s_{a_{i}}, i=1, \ldots, k$ (в частности $\left.s_{a} s_{b}=s_{b} s_{a}\right)$ u $s_{A}^{2} \Lambda=\Lambda$.

Полная последовательность является единицей.

Если $F$ - подграф $G, A$ - полная последовательность для $F$, то $s_{A} \Lambda$ отличается от $\Lambda$ направлением всех ребер, инцидентных $F u G \backslash F$.

Если $(A, B)$ - полная последовательность для $G$, то $s_{A}=s_{B}$.

Лемма 2. Отношение $\Lambda \sim \Lambda^{\prime}$, задаваемое равенством

$$
\exists A s_{A} \Lambda=\Lambda^{\prime},
$$

является отношением эквивалентности на множестве всех ориентачий графа.

Вершина $a$ называется (+)-вершиной ((-)-вершиной) в ориентированном графе $\Lambda$, если из нее не выходит (в нее не входит) ни одной стрелки. Пересечение множества всех 
(+)-вершин и множества всех (-)-вершин состоит из изолированных вершин. Объединение этих двух множеств состоит из так называемых (士)-вершин.

Последовательность $A$ называется (+)-последовательностью (соответственно (-)-последовательностью, $( \pm)$-последовательностью) в ориентации $\Lambda$, если $a_{1}-(+)$-вершина ((-)-вершина, ( \pm -вершина) в $\Lambda$ и $a_{i}-(+)$-вершина (соответственно (-)-вершина, (士)-вершина) в $s_{a_{i-1}} \ldots s_{a_{1}} \Lambda, i=2, \ldots, k$.

Нам удобно использовать термин цикл в гомологическом смысле: множество $c$ ребер называется циклом, если $\partial c=0$. Цикл называется простым, если каждая вершина цикла инцидентна не более чем паре его ребер (в частности, простым является нулевой цикл пустое множество ребер).

Два ребра простого цикла ориентированы однородно, если двигаясь вдоль цикла по направлению одной стрелки, мы достигаем второе ребро в его начале. Ориентация называется ациклической, если для любого простого цикла не все его ребра ориентированы однородно (иными словами, в графе нет контуров).

Лемма 3. ([1]) Следующие условия эквивалентны:

- ориентачия $\Lambda$ ачиклична,

- для $\Lambda$ существует полная (+)-единица,

- для А существует полная (-)-единица,

- для А существует полная (土)-единища.

Лемма 4. Если $A=\left\{a_{i}\right\}_{i=0}^{k}$ есть (士)-последовательность в ориентачии $\Lambda$, то $A^{\prime}=\left\{a_{k-i}^{k}\right\}_{i=0}^{k}$ есть $( \pm)$-последовательность в ориентачии $s_{A} \Lambda$.

Пусть $\Lambda$ - ачиклическая ориентачия, $A-( \pm)$-последовательность. Существует (+)-последовательность В и (-)-последовательность С такие, что

$$
s_{A}(\Lambda)=S_{B}(\Lambda)=s_{C}(\Lambda) .
$$

Доказательство. Первое утверждение очевидно. Для доказательства второго достаточно доказать следующее утверждение. Если $u-(-)$-вершина для ациклической ориентации $\Lambda$, то найдется (+)-последовательность $U$ такая, что $s_{U} \Lambda=s_{u} \Lambda$. Удалим вершину $u$, в качестве $U$ возьмем полную (+)-единицу оставшегося графа.

Лемма 5. Отношения $\sim_{+}, \sim_{-}, \sim_{ \pm}$такие, что

- $\Lambda \sim_{+} \Lambda^{\prime}$, если существует (+)-последовательность $A$, для которой $S_{A}(\Lambda)=\Lambda^{\prime}$,

- $\Lambda \sim \Lambda^{\prime}$, если существует (-)-последовательность $A$, для которой $S_{A}(\Lambda)=\Lambda^{\prime}$,

- $\Lambda \sim_{+} \pm \Lambda^{\prime}$, если существует $( \pm)$-последовательность А такая, что $S_{A}(\Lambda)=\Lambda^{\prime}$,

являются равносильными друг другу отношениями эквивалентности на множестве всех ачиклических ориентачий графа.

Отношение эквивалентности из предыдущей леммы будем обозначать $\sim_{\text {sgn }}$.

Циркуляцией простого цикла называется задание (одного из двух) направления последовательного обхода его ребер. Будем считать, что для каждого графа циркуляция каждого простого цикла фиксирована. 
Для простого цикла $c$ и ориентации $\Lambda$ интегралом $\oint_{c} d \Lambda$ называется разность числа ребер цикла $c$, направленных вдоль циркулящии, и числа ребер цикла $c$, направленных против циркуляции. Интеграл по нулевому циклу считается равным нулю. Отображение $c \rightarrow \oint_{c} d \Lambda$ обозначим $\oint d \Lambda$.

Если $B-$ базис (в $Z_{2}$-пространстве) циклов, состоящий из простых циклов, то сужение отображения $\oint d \Lambda$ на $B$ обозначим $\oint_{B} d \Lambda$.

Лемма 6. Существуют челье числа $k\left(G, B, c^{\prime}, c\right)$ и нечетное число $k\left(G, B, c^{\prime}\right)$ такие, что для всех $\Lambda$

$$
k\left(G, B, c^{\prime}\right) \oint_{c^{\prime}} d \Lambda=\sum_{c \in B} k\left(G, B, c^{\prime}, c\right) \oint_{c} d \Lambda .
$$

Доказательство. Нетривиален случай $c^{\prime} \notin B$. Для произвольных ориентаций $\Lambda, \Lambda^{\prime}$ и произвольного простого цикла $c$ определим вещественные функщии $\delta_{\Lambda}^{\Lambda^{3}}$ и $\delta_{C}^{\Lambda}$ на множестве ребер графа, полагая

$$
\begin{aligned}
i_{c}^{\Lambda}(l) & = \begin{cases}1, & \text { если ребро } l \in c \text { ориентировано вдоль циркуляции на } c, \\
0, & \text { если } l \notin c, \\
-1, & \text { если ребро } l \in c \text { ориентировано против ориентации на } c,\end{cases} \\
\delta_{\Lambda}^{\Lambda^{\prime}}(l) & = \begin{cases}1, & \text { если } l \text { ориентировано одинаково в } \Lambda \text { и } \Lambda^{\prime}, \\
-1, & \text { если } l \text { ориентировано различно в } \Lambda \text { и } \Lambda^{\prime} .\end{cases}
\end{aligned}
$$

Ранг системы $\left\{\delta_{c}^{\Lambda}\right\}_{c}$ равен $|B|$ (см. [3], теорема 6.7). Обозначим $n \rightarrow \bar{n}$ канонический эпиморфизм $\mathbf{Z} \rightarrow Z_{2}$. Система $\left\{\overline{i_{c}^{\Lambda}}\right\}_{c}$ состоит из характеристических (со значениями в $Z_{2}$ ) функщй системы $\{c\}_{c \in B}$ из $Z_{2}$-пространства циклов графа. Поэтому обе системы линейно независимы. Значит, система $\left\{i_{c}^{\Lambda}\right\}_{c \in B}$ линейно независима над $\mathbf{Z}$, а стало быть и над R. Итак,

Отсюда, для всех $l$

$$
\operatorname{rank}\left\{i_{c}^{\Lambda}\right\}_{c}=\operatorname{rank}\left\{i_{c}^{\Lambda}\right\}_{c \in B}
$$

$$
i_{c^{\prime}}^{\Lambda}(l)=\sum_{c \in B} \gamma\left(G, B, \Lambda, c^{\prime}, c\right) i_{c}^{\Lambda}(l)
$$

Коэффициенты $\gamma$ не зависят от $\Lambda$, так как $i_{c}^{\Lambda^{\prime}}=\delta_{\Lambda}^{\Lambda^{\prime}} i_{c}^{\Lambda}$. Эти коэффициенты рациональны. Избавившись от знаменателей, получаем, что для всех $\Lambda$ и $l$

$$
k\left(G, B, c^{\prime}, c\right)=\sum_{c \in B} k\left(G, B, c^{\prime}, c\right) i_{c}^{\Lambda}(j) .
$$

Коэффициенты $k$ есть целые числа, среди которых есть хотя бы одно нечетное. Переходя к суммированию по $l$, получаем, что для всех $\Lambda$

$$
k\left(G, B, c^{\prime}\right) \oint_{c^{\prime}} d \Lambda=\sum_{c \in B} k\left(G, B, c^{\prime}, c\right) \oint_{c} d \Lambda .
$$

Число $k\left(G, B, c^{\prime}\right)$ нечетно, так как в противном случае мы получили бы равенство

$$
0=\bar{k}\left(G, B, c^{\prime}\right) \overline{i_{c^{\prime}}^{\Lambda}}=\sum_{c \in B} \bar{k}\left(G, B, c^{\prime}, c\right) \overline{i_{c}^{\lambda}},
$$

в правой части которого не все коэффициенты равны нулю, что противоречит линейной независимости системы $\left\{\overline{i_{c}^{\Lambda}}\right\}_{c \in B}$. 
Следствие 1. Пусть $m$ - четное число. Если

$$
\oint_{B} d \Lambda \equiv \oint_{B} d \Lambda^{\prime} \quad(\bmod m)
$$

mo

$$
\oint d \Lambda \equiv \oint d \Lambda^{\prime} \quad(\bmod m)
$$

Теорема 1. Справедливы соотночения

$$
\Lambda \sim \Lambda^{\prime} \Longleftrightarrow \oint d \Lambda \equiv \oint d \Lambda^{\prime} \quad(\bmod 4) \Longleftrightarrow \oint_{B} d \Lambda \equiv \oint_{B} d \Lambda^{\prime} \quad(\bmod 4)
$$

и для ачиклических ориентачий

$$
\Lambda \sim_{\text {sgn }} \Lambda^{\prime} \Longleftrightarrow \oint d \Lambda=\oint d \Lambda^{\prime} \Longleftrightarrow \oint_{B} d \Lambda=\oint_{B} d \Lambda^{\prime} .
$$

Доказательство. Если $G$ - лес, то, как показано в [1], для любых его ориентаций $\Lambda \sim_{\text {sgn }}$ $\Lambda^{\prime}$ и тем более $\Lambda \sim \Lambda^{\prime}$. Поэтому будем далее предполагать, что $G-$ не лес. В обоих случаях импликации етривиальны. Справедливость противоположных импликаций во втором случае вытекает из следствия 1 при $m=4$ и $m=0$.

Докажем, что

$$
\Lambda \sim \Lambda^{\prime} \Longrightarrow \oint d \Lambda \equiv \oint d \Lambda^{\prime} \quad(\bmod 4)
$$

Достаточно найти ориентацию $\Delta \sim \Lambda$, отличающуюся от $\Lambda^{\prime}$ на меньшее число ребер, чем $\Lambda$ от $\Lambda^{\prime}$. Отметим в $G$ ребра, на которых $\Lambda \neq \Lambda^{\prime}$ и удалим их. В полученном графе $G^{*}$ не может быть компоненты связности, содержащей концы одного из отмеченных ребер, так как в противном случае, двигаясь в графе $G$ по этой стрелке и стрелкам графа $G^{*}$, принадлежащим данной компоненте, можно было бы получить простой цикл $c$, в котором отмечено ровно одно ребро и оно ориентировано по-разному в ориентациях $\Lambda$ и $\Lambda^{\prime}$, и следовательно,

$$
\left|\oint_{c} d \Lambda-\oint_{c} d \Lambda^{\prime}\right|=2
$$

Поэтому найдется компонента $F$, для которой все отмеченные ранее ребра, инцидентные ей (как подграфу графа $G$ ), соединяют $F$ и $G \backslash F$. Пусть $A$ - полная последовательность для $F$. Поэтому $\Delta=s_{A} \Lambda-$ искомая ориентация.

Доказательство того, что

$$
\Lambda \sim_{\text {sgn }} \Lambda^{\prime} \Longrightarrow \oint d \Lambda=\oint d \Lambda^{\prime}
$$

мы воспроизведем из депонированной статьи [2] (с некоторым обобщением). Достаточно найти ориентацию $\Delta \sim_{\text {sgn }} \Lambda$, отличающуюся от $\Lambda^{\prime}$ на меньшее число ребер, чем $\Lambda$ от $\Lambda^{\prime}$. Отметим в $G$ ребра, на которых $\Lambda \neq \Lambda^{\prime}$, и удалим их. В полученном графе $G^{*}$ зафиксируем компоненты связности, которым инцидентно хотя бы одно отмеченное ребро. Среди этих компонент найдется компонента $F$, для которой все стрелки, инцидентные ей (как подграфу графа $G$ ), направлены от $G \backslash F$ к $F$ в ориентации $\Lambda$, так как иначе для каждой компоненты нашлась бы стрелка с началом в этой компоненте и, двигаясь в графе $G$ по этим стрелкам и стрелкам графа $G^{*}$, мы получили бы простой цикл, в котором 
все отмеченные ребра ориентированы однородно в ориентации $\lambda$, а в ориентации $\Lambda^{\prime}$ они ориентированы также однородно, но в другом направлении. Отсюда

$$
\oint d \Lambda \neq \oint d \Lambda^{\prime}
$$

Пусть $A$ - полная (+)-единица для $\left(F,\left.\Lambda\right|_{F}\right)$. Все ребра, инцидентные $F$, награвлены к $F$ в ориентации $\Lambda$, поэтому $A-(+)$-последовательность в $\Lambda$, и $\Delta=s_{A} \Lambda$ - искомая ориентация.

\section{Список литературы}

1. Бернштейн И. Н., Гельфанд И. М., Пономарев В. А., Функторы Кокстера и теорема Габриеля. Успехи матем. наук (1973) 28, №2, 19-33.

2. Колмыков В. А., Меньших В. В., Субботин В. Ф., Сумин М. В., Некоторые свойства ациклических графов и связанных с ними многочленов. Деп. ВИНИТИ 12.12.83, №6707-83.

3. Свами М., Тхуласираман К., Графы, сети и алгоритмы. Мир, Москва, 1984.

Статья поступила 23.06.2000. 